\section{The Crystal Structure of $\mathrm{Cs}_{2} \mathrm{Ga}_{2} \mathrm{~S}_{5}$}

\author{
Daniel Friedrich, ${ }^{[\mathrm{a}]}$ Arno Pfitzner, ${ }^{[\mathrm{a}]}$ and \\ Marc Schlosser*[a]
}

Keywords: Crystal structure; caesium thiogallate.

The rod-like crystals were synthesized by thermal decomposition of a non-stoichiometric tablet of $\mathrm{CsN}_{3}, \mathrm{GaS}$ and $\mathrm{S}$ in an evacuated quartz glass ampoule.

The crystal structure of the solid $\mathrm{Cs}_{2} \mathrm{Ga}_{2} \mathrm{~S}_{5}$ was determined by single crystal X-ray diffraction analysis. The compound crystallizes monoclinic in the space group $C 2 / c$ (no. 15) with the unit-cell dimensions $a=12.762(3) \AA, b=7.208(1) \AA, c=$ 12.495(3) $\AA$, $\beta=108.29(3)^{\circ} ; \mathrm{Z}=4$.

The main structural feature of $\mathrm{Cs}_{2} \mathrm{Ga}_{2} \mathrm{~S}_{5}$ is a one-dimensional polyanionic chain of $\mathrm{GaS}_{4}$ tetrahedra in a matrix of caesium atoms. The chains contain disulfide ions similar to the parent compound $\mathrm{CsGaS}_{3}{ }^{[1]}$. In $\mathrm{Cs}_{2} \mathrm{Ga}_{2} \mathrm{~S}_{5}$, gallium is coordinated to three sulfide ions and one disulfide ion.

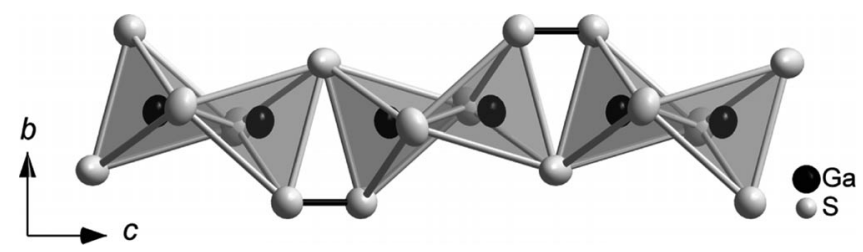

Figure 1. Projection of the anionic chain structure of $\mathrm{Cs}_{2} \mathrm{Ga}_{2} \mathrm{~S}_{5}$ along [100].

[1] M. Suseela Devi, K. Vidyasagar, J. Chem. Soc., Dalton Trans. 2002, 4751-4754.

\section{Analyse von Spurenelementen in Kristallen mittels ETV ICP OES}

\author{
Rainer Bertram*[a]
}

Keywords: Kristalle, chemische Analyse, ETV-ICP OES

Die ICP OES-Emissionsspektrometrie ist eine der wichtigsten Techniken der instrumentellen Analytik. Mitunter ist der Gehalt an Spurenelementen in den Aufschlusslösungen (Mikrowellenaufschluss mit Säuren) aber unterhalb der Bestimmungsgrenzen. Zur direkten Spurenanalyse fester Proben wurde das ICP OES-Spektrometer mit einer ETV-Einheit (elektrothermische Verdampfung) gekoppelt. ${ }^{[1,2]}$

Bei der Analyse von SiC-Einkristallen kann die Kalibrierung sowohl mit eingetrockneten Elementstandardlösungen als auch über matrixgleiche Feststoffe (Referenzmaterialien) erfolgen. Die SiC-Matrix verdampft selbst nicht und gelangt somit auch nicht in das ICP-Plasma. ${ }^{[1,2]}$ Für die Elemente Al, Fe, Ti, V und $\mathrm{W}$ wurde eine Anreicherung hin zum Kristallende gefunden, wogegen $\mathrm{Cu}, \mathrm{Cr}, \mathrm{Mg}, \mathrm{Mn}$ und $\mathrm{Zr}$ homogen im SiCKristall verteilt sind. ${ }^{[2]}$

Der Mg-Gehalt in ZnO-Einkristallen kann durch Lösungsanalyse bestimmt werden und dieser dient dann als Referenzwert für die Ergebnisse, die mit der direkten Feststoffmethode (ETV ICP OES) erhalten wurden. Im Unterschied zum SiC wird das $\mathrm{ZnO}$ bei der thermischen Verdampfung vollständig in das ICPPlasma überführt, was zu einer erheblichen Matrixbelastung des Plasmas führt. Die Ergebnisse zeigen, dass trotz Verwendung von verschiedenen Kalibrationsverfahren meist ein Minderbefund an $\mathrm{Mg}$ - im Vergleich zur Lösungsanalyse - ermittelt wurde.

[1] R. Matschat, J. Hassler, H. Traub, A. Dette, Anal. Bioanal. Chem. 2005, 383, 1060-1074.

[2] R. Bertram, H. Stephanowitz, H.-J. Heinrich, H.-J. Rost, C. Lüdge, J. Hassler, Freiberger Forschungsheft 2006, B337, 122-138.
* Dr. M. Schlosser

E-Mail: Marc.Schlosser@chemie.uni-regensburg.de

[a] Institut für Anorganische Chemie

Universität Regensburg

Universitätsstraße 31

93040 Regensburg, Germany

\footnotetext{
* Dr. R. Bertram

E-Mail: rainer.bertram@ikz-berlin.de

[a] Leibniz-Institut für Kristallzüchtung

Max-Born-Str. 2

12489 Berlin, Germany
} 